\title{
A Mutual Projection based Proportional Features Selection for Face Identification
}

Singaravelan. S

PSR Engineering College, Sivakasi

India

singaravelan.msu@gmail.com

Murugan. D

Manonmaniam Sundaranar University

Tirunelveli

India

ABSTRACT: A analysis of human facial images has become increasingly important due to its numerous applications. In this regards, extracting facial parameter is vital and various studies have been done in this field. Hence in our proposed work, first time up to our knowledge, a robust automatic method is introduced for determining facial angles from profile view images using radon transform. Radon transform is a kind of linear integration along a specific direction and angles play an important role to do this transform. The global features were rather considered by constructing a linear discriminant analysis (LDA) and also local features were rather considered by locality preserving projection (LPP). Our proposed combined algorithm has not only good precision, but also efficient performance and robust with noisy, scale and rotated image environments. In this work, several experiments have been conducted to analyze the robustness of our proposed Radon Combined Global and Local Preserving Features (RCGLPF) algorithm along with other existing conventional algorithms.

Keywords: Radon Transform, Linear Discriminant Analysis, Locality Preserving Projection, Combined Features, RCGLPF

Received: 13 September 2018, Revised 2 November 2018, Accepted 9 November 2018

DOI: $10.6025 / \mathrm{jmpt} / 2019 / 10 / 1 / 27-39$

(C) 2019 DLINE. All Rights Reserved

\section{Introduction}

Now a days, the importance of human facial images analysis has significantly increased due to its numerous applications, including facial surgery analysis human face plays an important role in appearance of person. Face recognition is a very difficult problem due to a substantial variation in light direction ( illumination), different face poses, diversified facial expressions, ageing (changing the age over time) and occlusions ( like glasses, hair, cosmetics). So the building of automated systems that accomplishes such objectives is very challenging. In the last decades many system with recognition rate greater than $90 \%$ has 
been done however a perfect system with $100 \%$ recognition rate remains challenge.

Face recognition algorithms are classified into the following categories.

1. Comprehensive methods: whole image as input and extract the features.

2. Feature based methods: Local facial features to recognize a face.

3. Hybrid methods: both feature based and comprehensive features to recognize a face. These methods have the potential to offer better performance than individuals.

In recent years, considerable progress has been made in the area of face recognition with the development of many techniques. Whilst these techniques perform extremely well under constrained conditions, the problem of face recognition in uncontrolled noisy environment remains unsolved. During the transmission of images over the network, some random usually unwanted variation in brightness or color information may be added as noise. The previous study proposed several noise removal filtering algorithms. Most of them assume certain statistical parameters and know the noise type a priori, which is not true in practical cases. Applying a filtering algorithm that is sensitive to additive noise to an image that has been degraded by a multiplicative noise doesn't give an optimal solution. Also the difficulty in removing salt/pepper noise from binary image is due to the fact that image data as well as the noise share the same small set of values (either 0 or 1 ) which complicates the process of detecting and removing the noise. This is different from grey images where salt/pepper noise could be distinguished as pixels having big difference in grey level values compared with their neighborhood. Many algorithms have been developed to remove salt/pepper noise in document images with different performance in removing noise and retaining fine details of the image.

Invariance to rotation and scale are desirable in many practical applications. One important application is image classification and retrieval where one wants to classify or retrieve images having the same content but at different orientation and scale. For instance, in content based image retrieval (CBIR), images are retrieved from a database using features that best describe the orientation and scale of objects in the query image.

The Radon transform (RT) has a long history of application in computer vision and face recognition and relatively recently has been applied in a variety of image processing problems. Hence in our proposed work, first time up to our knowledge such an face recognition application is presented in where the Radon transform and LDA and LPP is used to create features used by the recognition.

From an appearance representation standpoint, Principal Component Analysis (PCA) Multidimensional Scaling (MDS), Linear Discriminant Analysis (LDA), and Locality Preserving Projections (LPP) based techniques are more relevant. In those appearance based face recognition, the global features preserving techniques namely PCA, MDS, and LDA effectively preserves the Euclidean structure of face space or the global features. On the other hand, the local feature preservation technique namely Locality Preserving Projections (LPP) preserves local information and obtains a face subspace that best detects the essential face manifold structure. Global features preserving techniques suffer when the noises affect the global features like the structure of the facial images, while local features preserving techniques suffer when the image noises affect the local intensity pixels.

The rest of the paper is organized as follows: Section 2 describes various previous works. Material and Methods discussed in section 3. Proposed Radon Combined Global and Local Preserving Features (RCGLPF) concepts given in Section 4. In Section 5 , the experimental results have been discussed with respect to percentage of correct recognition considering ORL facial image database under various noisy, scaled and rotated environments for RCGLPF in comparison with other traditional RT, PCA, LDA and LPP algorithms. The paper is completed with some final remarks in section 6.

\section{Previous Works}

Sambhunath Biswas [1] proposed two different algorithms with two different kinds of features and these features are reasonably robust against illumination variation and orientation. These facts are supported by experimental results based on images of Yale and ORL data bases respectively $96 \%$ recognition rate has been achieved using the proposed algorithm.

Nicolas[2] expression recognition scheme based on features derived from the optical flow between a neural and a face at a 'apex' of the expression has presented, utilized radon transform to estimate the basic movement within the face area, $87.5 \%$ recognition

\begin{tabular}{lllllll}
\hline 28 & Journal of Multimedia Processing and Technologies & Volume & 10 & Number & 1 & March 2019 \\
\hline
\end{tabular}


rate has been achieved.

Belhumeur[3] work is compared two methods eigen faces and fishers faces features are computed, $83 \%$ recognition rate hasbeen achieved.

Bilal [4] approach to face recognition with Radon Discrete Wavelet Transform is presented. The method uses Radon Discrete Wavelet Transform for both finding feature points and extracting feature vectors. From the experimental results, 94.48\% recognition rate has been achieved.

Rerkchai [5] have presented a highly robust method for face authentication. Techniques introduced in this work are composed of two stages. Firstly, the feature of face is to be detected by the principle of Trace Transform. Then, in the second stage, the Hausdorff distance and modified Shape Context are employed to measure and determine of similarity between models and test images. From the experimental result of 6,325 images, the average of accuracy rate is higher than $87 \%$.

Hiremath[6] a novel method for three dimensional (3D) face recognition using Radon transform and Symbolic LDA based features of 3D range face images is proposed. In this method, the Symbolic LDA based feature computation takes into account face image variations to a larger extent and has advantage of dimensionality reduction. The experimental results have yielded $99.50 \%$ recognition rate has been achieved.

Appati[7] observed that Radon Transform based feature extraction for face recognition (RTFR) yielded a good recognition rate though only four features were extracted from the face for recognition purpose. $95 \%$ recognition rate has been achieved.

Zhao [8] presented face recognition systems which combines PCA and LDA, 93.3\% recognition rate has been achieved.

\section{Proposed Method}

The method used in this paper for face recognition is depicted in figure (1). It consists the enrollment phase and the testing phase. In the enrollment phase the training set of images are transformed into Radon space using the Radon Transform. The data generated by Radon Transform are reduced using Principal Components Analysis (PCA). From those reduced data set (i.e. the most effective components) a set of basis vectors which maximizes the ratio between-class scatter and within-class scatter using Linear Discrimenant Analysis (LDA) to preserving global features. Locality preserving projection applied to basis vectors to preserving local features. Adding local features to global features and constructing the one feature weight vector for per classes. And store it to the all classes weight vector in feature weight vector database. The length of the basis vector depends on the number of components selected by the PCA stage. In the testing phase the input image is transformed into the Radon space using that set of directions which were used in the enrollment phase. The PCA reduction is carried out as same as in the

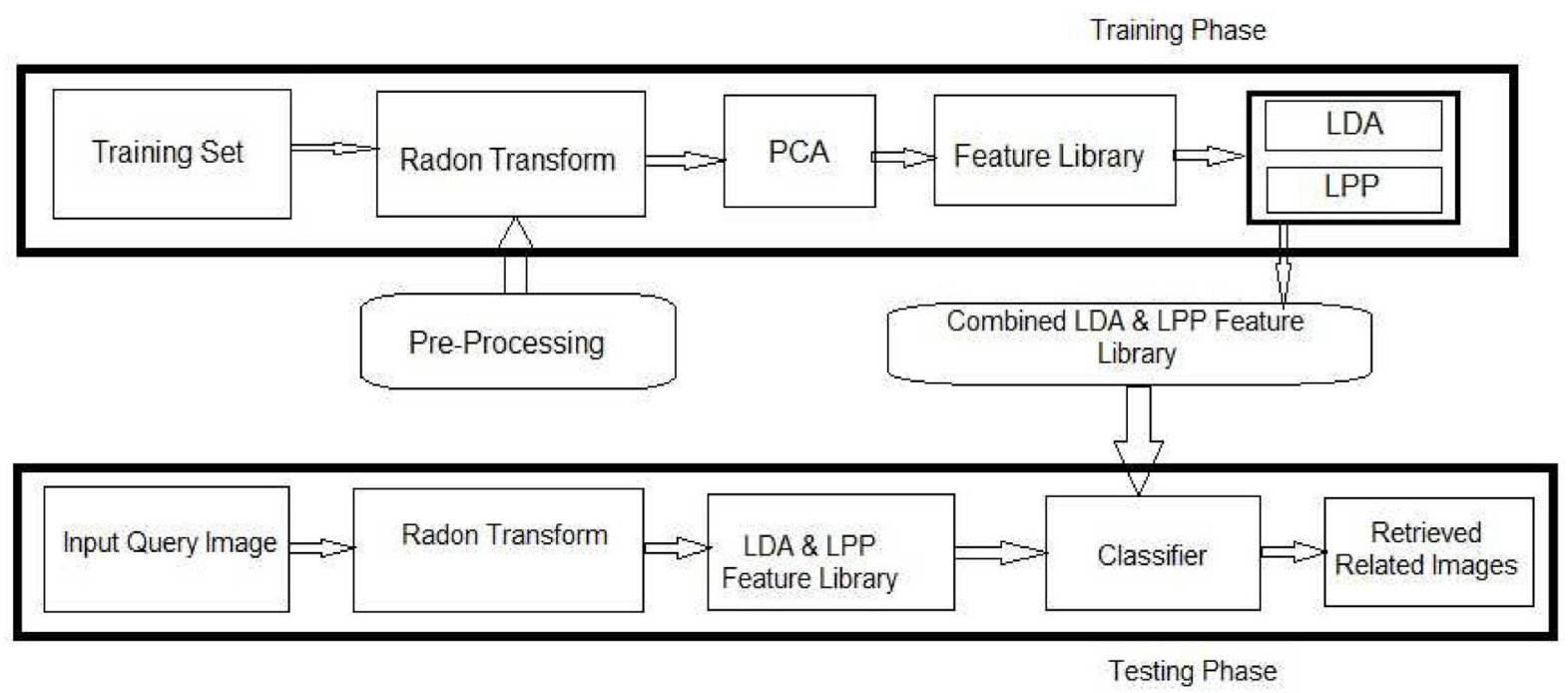

Figure 1. Proposed CBIR Method 
enrollment phase. The resultant vector is projected into the basis vectors stored in the data base that is by inner product method. The method which is stated in this paper was evaluated using the ORL data base which contains photographs of faces taken at the Olivetti Research Laboratory in Cambridge between April 1992 and April 1994. There are 10 different images of 40 distinct subjects so there are 400 images in the data base. The images are grayscale with a resolution of $92 \times 112$. In the following sections the discussion of each main part of the method of process is presented.

\section{Radon Transform}

The radon transform is used many applications like categories the visual objects [12], rotation invariants in objects identification $[13,15]$, face recognitions [14], image recognitions [16]. The radon transform is the projection of the image intensity along a radial line oriented at a specific angle. The radial coordinates are the values along the $x$ '-axis, which is oriented at $r$ degree counter clockwise from the x-axis. The origin of the both axis is the center pixel of the image. Radon transform of an image representation as a matrix, called sinogram.

\section{Horizontal and Vertical Projection}
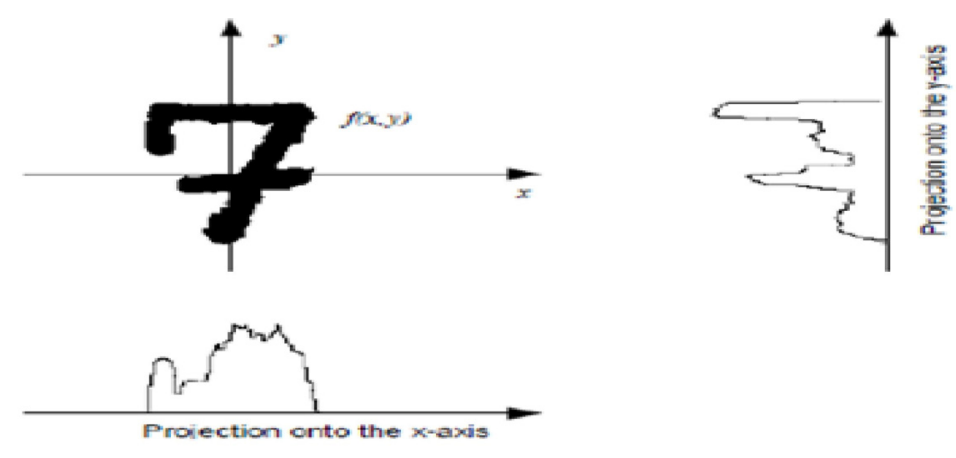

Figure 2. Radon Transform Horizontal \& Vertical Projection View

The popular form expresses lines is

$$
t=x * \cos (\theta)+y^{*} \sin (\theta)
$$

$\theta$ angle $\& t-$ smallest distance to the origin of the coordinate system.

Projection can be computed along any angle $\mathrm{r}$, by use general equation of the radon transform.

$$
g(t, \theta)=\iint_{-\infty}^{+\infty} f(x, y) \delta(t-x(\cos (\theta)-y \sin (\theta))) d x d y
$$

The very strong property of the radon transform is the ability to extract lines (curves in general) from very noisy images.

\section{Rotation Invariance}

The radon transform of a two variable function $x$ is defined as

$$
R_{\theta} x(t)=\int_{-\infty}^{\infty} x(t \cos \theta-s \sin \theta, t \sin \theta+s \cos \theta) d s
$$

where $(t, \theta) \in(-\infty, \infty) \times(0, \pi) \forall s \in R$

To compute the value at any given point $(\mathrm{t}, \theta)$ in the radon domain by integrating along the line:

$$
(u, v)=(t \cos \theta-s \sin \theta+s \cos \theta)
$$

If $x^{6}$ is a rotated copy of $x$ by on angle $\theta$

\begin{tabular}{lllllll}
\hline $30 \quad$ Journal of Multimedia Processing and Technologies & Volume & 10 & Number 1 & March 2019 \\
\hline
\end{tabular}


Simple proof shows $R_{\theta} \bar{x}(t)=R_{\theta+\hat{\theta}} x(t) \forall t, \theta$

Let $\sigma_{\theta} \triangleq \operatorname{Var}_{t}\left[R_{\theta} x(t)\right]$ denotes the variance with respect to $t$ of the $R T$ at $\theta$.

$\sigma_{\theta}$ - Useful in estimating the principal orientation in an image.

Estimate $\hat{\theta}$

$$
\hat{\theta}=\arg \min _{\theta}\left(\frac{d^{2} \sigma \theta}{d \theta^{2}}\right)
$$

\section{Scale Invariance}

Let $x$ ' is the scaled copy of $x$ with scaling factor $\varepsilon$ such that

$$
\bar{x}(u, v)=x(\xi u, \xi v)
$$

Relate this to $R T$,

$$
\begin{aligned}
R_{\theta} \bar{x}(t) & =\int_{-\infty}^{\infty} \bar{x}(t \cos \theta-s \sin \theta+s \cos \theta) d s \\
& =\int_{-\infty}^{\infty} x(\xi t \cos \theta-\xi s \sin \theta, \xi t \sin \theta+\xi s \cos \theta) d s \\
& =\frac{1}{\xi} R_{e} x(\xi t)
\end{aligned}
$$

From this observation, scale invariance can be achieved through the normalization in the $R T$.

\section{Principal Component Analysis (PCA)}

Principal Components Analysis (PCA) is one of several statistical tools available for reducing the dimensionality of a data set $[19,20$, and 24]. Its relative simplicity and both computational and in terms of understanding what's happening and make it a particularly popular tool. The major goal of principal components analysis is to reveal hidden structure in a data set. In so doing, we may be able to identify how different variables work together to create the dynamics of the system.

- Reduce the dimensionality of the data

- Decrease redundancy in the data

- Filter some of the noise in the data

- Compress the data

The training set $D$ and testing set $Q$ on basic vectors:

Step 1: Calculate mean value $m$ for training set and subtract it from the training set

$$
m=\frac{1}{n} \Sigma_{i=1}^{n} D_{i}
$$

All training set $D_{i}=D_{i}-m$

Step 2: Calculate the eigenvectors and eigenvalues of the training set covariance matrix $C_{t}$

$$
C_{t}=D \cdot D^{Q}
$$


The eigenvectors corresponding $N$ largest eigenvalues of $C_{t}$ These construct the principal component matrix $(V)$.

Step 3: The basic vector matrix $(E)$ is defined by:

$$
E=V \cdot D^{Q}
$$

Step 4: The reduced feature vectors for training and testing data calculated by:

$$
\begin{aligned}
& W D=D . E \\
& W Q=Q . E
\end{aligned}
$$

\section{Linear Discriminant Analysis (LDA)}

Linear Discriminant Analysis easily handles the case where the within-class frequencies are unequal and their performances have been examined on randomly generated test data. This method maximizes the ratio of between-class variance to the withinclass variance in any particular data set thereby guaranteeing maximal separability. The use of Linear Discriminant Analysis for data classification is applied to classification problem in face recognition. We decided to implement an algorithm for LDA in hopes of providing better classification compared to Principal Components Analysis. Data sets can be transformed and test vectors can be classified in the transformed space by two different approaches.

Class-independent Transformation: This approach involves maximizing the ratio of overall variance to within class variance. This approach uses only one optimizing criterion to transform the data sets and hence all data points irrespective of their class identity are transformed using this transform.

Class-dependent Transformation: This type of approach involves maximizing the ratio of between class variance to within class variance. The main objective is to maximize this ratio so that adequate class separability is obtained.

\section{Preserving Global Features in LDA Domain}

Formulate the data sets and the test sets, which are to be classified in the original space.[26]

- Compute the mean of each data set and mean of entire data set.

- Mean $($ entire data set $)=$ Class Probabilities X Mean value of each classes.

- In LDA, within-class and between-class scatter are used to formulate criteria for class separability. Within-class scatter is the expected covariance of each of the classes. The scatter measures are computed using Equations.

$$
S_{w}=\sum_{j} p_{j} \times\left(\operatorname{cov}_{j}\right)
$$

The between-class scatter is computes using the following equation

$$
S_{b}=\sum_{j}\left(\mu_{j}-\mu_{3}\right) \times\left(\mu_{j}-\mu_{3}\right)^{T}
$$

- Note that can be thought of as the covariance of data set whose members are the mean vectors of each class. As defined earlier, the optimizing criterion in LDA is the ratio of between-class scatter to the within-class scatter. The solution obtained by maximizing this criterion defines the axes of the transformed space. It should be noted that if the LDA is a class dependent type, for $L$-class separate optimizing criterion are required for each class. The optimizing factors in case of class dependent type are computed as

$$
\text { criterion }_{j}=\operatorname{inv}\left(\operatorname{cov}_{j}\right) \times S_{b}
$$

- For any $L$-class problem we would always have $L-1$ non-zero eigen values. The eigen vectors corresponding to non-zero eigen

\begin{tabular}{llllll}
\hline 32 & Journal of Multimedia Processing and Technologies \\
\hline
\end{tabular}


values for the definition of the transformation. The decision region in the transformed space is a solid line separating the transformed data sets thus, For the class dependent LDA,

$$
\text { transformed_set_j } \text { transform }_{-} j^{T} \times \text { set_j }
$$

Similarly the test vectors are transformed and are classified using the Euclidean distance of the test vectors from each class mean.

- Once the transformations are completed using the LDA transforms, WLDA is found as the Eigen vector matrix of the different criteria Equation1.

\section{Locality Preserving Projections (LPP)}

LPP finds an embedding that preserves local information[25], and obtains a face subspace that best detects the essential face manifold structure. To be specific, the manifold structure is modeled by a nearest- neighbor graph which preserves the local structure of the image space. A face subspace is obtained by Locality Preserving Projections (LPP).PCA and LDA aim to preserve the global structure. However, in many real world applications, the local structure is more important, Locality Preserving Projection (LPP) a new algorithm for learning a locality preserving subspace, LPP seeks to preserve the intrinsic geometry of the data and local structure. The objective function of LPP is as follows:

$$
\min \sum_{i j}\left(y_{i}-y_{j}\right)^{2} S_{i j}
$$

The transformation vector $\boldsymbol{w}$ that minimizes the objective function is given by the minimum eigenvalue solution to the generalized eigenvalue problem:

$$
X L X^{T} w=\lambda X D X^{T} w
$$

Note that the two matrices $X L X_{T}$ and $X D X_{T}$ are both symmetric and positive semi-definite, since the Laplacian matrix $L$ and the diagonal matrix $D$ are both symmetric and positive semi-definite.

\section{Adding Local Features:}

- LPP Projection: The local features are added to the preserved global features in order to increase the robustness of our technique against various noises. Actually the local features preserving technique seeks to preserve the intrinsic geometry of the data and local structure. The following are the steps to be carried out to obtain the Laplacian transformation matrix $W_{L P P}$, which we use to preserve the local features.

- Constructing the nearest-neighbor graph: Let G denote a graph with $n$ nodes. The $i$ th node corresponds to the face image $x i$. We put an edge between nodes $i$ and $j$ if $\boldsymbol{x} i$ and $\boldsymbol{x} j$ are "close", i.e. $\boldsymbol{x} i$ is among $k$ nearest neighbors of $\boldsymbol{x} i$ or $\boldsymbol{x} i$ is among $k$ nearest neighbors of $x \boldsymbol{j}$. The constructed nearest neighbor graph is an approximation of the local manifold structure. Note that, here we do not use the $\varepsilon$ - neighborhood to construct the graph. This is simply because it is often difficult to choose the optimal $\varepsilon$ in the real world applications, while $k$ nearest neighbor graph can be constructed more stably. The disadvantage is that the $k$ nearest neighbor search will increase the computational complexity of our algorithm. When the computational complexity is a major concern, one can switch to the $\varepsilon$ - neighborhood.

- Choosing the Weights: If node $i$ and $j$ are connected, put

$$
S_{i j}=e^{-\frac{\left\|x_{i}-x_{j}\right\|^{2}}{t}}
$$

where $t$ is a suitable constant. Otherwise, put $S_{i j}=0$. The weight matrix $S$ of graph $G$ models the face manifold structure by preserving local structure.

- Eigenmap: Compute the eigenvectors and eigenvalues for the generalized eigenvector problem: 


$$
X L X^{T} w=\lambda X D X^{T} w
$$

where $D$ is a diagonal matrix whose entries are column (or row, since $S$ is symmetric) sums of $S, D i i=\Sigma j S j i . L=D-S$ is the Laplacian matrix. The $i t h$ row of matrix $X$ is $x i$. Let $w 0, w 1, \ldots, w k-1$ be the solutions of equation (1), ordered according to their eigenvalues, $0 \leq \lambda 0 \leq \lambda 1 \leq L \leq \lambda k$-1. These eigenvalues are equal to or greater than zero, because the matrices $X L X T$ and $X D X T$ are both symmetric and positive semi-definite. By considering the transformation space WLDA and WLPP, the embedding is done as follows:

$$
\begin{aligned}
& x \rightarrow y=W T x \\
& W=W L D A W L P P \\
& W L P P=[\boldsymbol{w} 0, \boldsymbol{w} 1, \ldots, \boldsymbol{w} k-1]
\end{aligned}
$$

where $y$ is a $k$-dimensional vector. $W L D A, W L P P$ and $W$ is the transformation matrix of $L D A, L P P$ and $R C G L P F$ algorithms respectively.

\section{Experimental Results and Discussion}

The proposed Face Recognition (RCGLPF) was applied in Our Database of Faces, (formerly 'The ORL Database of Faces'), contains a set of face images, ten different images of each of 40 distinct subjects. For some subjects, the images were taken at different times, varying the lighting, facial expressions (open / closed eyes, smiling / not smiling) and facial details (glasses / no glasses). All the images were taken against a dark homogeneous background with the subjects in an upright, frontal position (with tolerance for some side movement). Totally four experiments conducted. Each with different query environments like normal, rotated, scaled and noisy and performance was evaluated and represent in Tables $(1,2,3,4)$. The two types of distance measure function was used in our proposed method is L1 \& L2 performance was evaluated.The proposed method overall performance was compared to other existing traditional methods in Figure 5.

\section{Situation-1 :}

In this situation performance measured faces in different rotated angle in query image. The selected Query face rotated angles was $30^{\circ}, 60^{\circ}, 90^{\circ}, 120^{\circ}, 150^{\circ}$ and $180^{\circ}$. See Table (1). It obtained percentage is around $98.7 \%$.

\section{Situation - 2:}

In this situation performance measured faces in different scaling factor in query image The selected Query face scaling factor was 30x30, 60x60,90x90 and 120x120. See Table (2). It obtained percentage is around 98.9\%.

\section{Situation -3 :}

In this situation performance measured face in standard pose used as query image. See Table (3). It obtained percentage is around $98.8 \%$.

\section{Situation - 4:}

In this situation performance measured face in different noise information added to query image like salt \& pepper, additive and uniform noise in query image. See Table (4). It obtained percentage is around 97.5\%.

\begin{tabular}{|l|c|c|c|c|c|c|}
\hline \multicolumn{7}{|c|}{ Angle in Degrees } \\
\hline Method & $\mathbf{3 0}$ & $\mathbf{6 0}$ & $\mathbf{9 0}$ & $\mathbf{1 2 0}$ & $\mathbf{1 5 0}$ & $\mathbf{1 8 0}$ \\
\hline PCA & 56.8 & 67.3 & 61.5 & 68.0 & 69.5 & 66.0 \\
\hline LDA & 78.6 & 76.3 & 73.0 & 75.4 & 74.8 & 71.3 \\
\hline LPP & 82.4 & 79.0 & 76.0 & 80.0 & 83.5 & 79.0 \\
\hline RT & 79.8 & 81.2 & 79.8 & 84.7 & 81.8 & 82.0 \\
\hline RCGLPF & 97.8 & 96.0 & 98.2 & 98.7 & 97.2 & 97.5 \\
\hline
\end{tabular}

Table 1. Query Face in Different Angles

$34 \quad$ Journal of Multimedia Processing and Technologies Volume 10 Number 1 March 2019




\begin{tabular}{|l|l|l|l|l|c|}
\hline \multicolumn{6}{|c|}{ Scale in Sizes } \\
\hline Method & $\mathbf{3 0 \times 3 0}$ & $\mathbf{6 0 x 6 0}$ & $\mathbf{9 0 x 9 0}$ & $\mathbf{1 2 0 \times 1 2 0}$ & $\mathbf{1 5 0 \times 1 5 0}$ \\
\hline PCA & 63.2 & 67.8 & 69.0 & 71.5 & 73.8 \\
\hline LDA & 68.8 & 71.4 & 73.6 & 77.2 & 79.7 \\
\hline LPP & 70.0 & 73.6 & 76.8 & 79.3 & 83.5 \\
\hline RT & 73.5 & 77.0 & 79.2 & 84.5 & 88.9 \\
\hline RCGLPF & 88.7 & 93.7 & 95.0 & 97.9 & 98.9 \\
\hline
\end{tabular}

Table 2. Query Face in Different Scales

\begin{tabular}{|l|l|l|l|l|l|}
\hline \multicolumn{5}{|c|}{ Recognition Rate } \\
\hline Class & PCA & LDA & LPP & RT & RCGLPF \\
\hline Person 1 & 68.0 & 72.8 & 80.4 & 86.2 & 96.8 \\
\hline Person 2 & 65.7 & 74.2 & 78.2 & 81.5 & 93.5 \\
\hline Person 3 & 67.3 & 73.0 & 79.2 & 83.6 & 94.8 \\
\hline Person 4 & 69.3 & 76.9 & 83.5 & 88.0 & 98.0 \\
\hline Person 5 & 70.3 & 75.0 & 81.5 & 86.0 & 98.8 \\
\hline
\end{tabular}

Table 3. Query Face in Different Persons

\begin{tabular}{|c|c|c|c|c|c|}
\hline \multirow[b]{2}{*}{ Noise } & \multicolumn{4}{|c|}{ Recognition Rate } & \multirow[b]{2}{*}{ RCGLPF } \\
\hline & PCA & LDA & LPP & RT & \\
\hline Salt \& Pepper Noise & \multicolumn{5}{|c|}{ V-Variance } \\
\hline $\mathrm{V}=0.5$ & 66.9 & 96.04 & 97.64 & 96.45 & 99.40 \\
\hline $\mathrm{V}=0.1$ & 64.7 & 89.57 & 90.7 & 92.53 & 94.25 \\
\hline $\mathrm{V}=0.15$ & 60.02 & 94.0 & 94.72 & 96.34 & 97.20 \\
\hline $\mathrm{V}=0.2$ & 56.77 & 76.25 & 76.72 & 81.67 & 88.75 \\
\hline Additive Noise & \multicolumn{5}{|c|}{ V-Variance, M - Mean } \\
\hline $\mathrm{V}=0.5 \quad \mathrm{M}=0.5$ & 60.20 & 83.23 & 84.45 & 86.45 & 91.50 \\
\hline $\mathrm{V}=0.1 \quad \mathrm{M}=0.1$ & 44.75 & 53.80 & 55.57 & 61.56 & 88.45 \\
\hline $\mathrm{V}=0.15 \quad \mathrm{M}=0.15$ & 34.37 & 41.80 & 43.00 & 40.34 & 75.58 \\
\hline $\mathrm{V}=0.2 \quad \mathrm{M}=0.2$ & 22.62 & 25.90 & 26.95 & 30.25 & 72.60 \\
\hline Uniform Noise & \multicolumn{5}{|c|}{ V-Variance } \\
\hline $\mathrm{V}=0.5$ & 68.51 & 95.10 & 96.92 & 97.50 & 98.30 \\
\hline $\mathrm{V}=0.1$ & 66.12 & 93.88 & 94.17 & 96.12 & 99.20 \\
\hline $\mathrm{V}=0.15$ & 63.72 & 90.10 & 90.20 & 89.55 & 92.10 \\
\hline $\mathrm{V}=0.2$ & 61.57 & 82.95 & 83.62 & 84.87 & 88.90 \\
\hline
\end{tabular}

Table 4. Query Face in Different Noises 


\section{Discussions}

Face recognitions using our proposed method Radon Combined Global and Local Preserving Features (RCGLPF). This proposed methods recognition rate is compared with the mention literatures \& references $[2,4,6,9$, and 10$]$. This is represent our proposed combined feature space matrix is very effectively represent the face features. From the different situation conducted in different query environments it appears that the performance is improved with the increased number of related faces retrieved followed by query image. Table [1,2,3, and 4] represented the different query types used and achieved the better recognition rate. Fig [5] represented the proposed method recognition rates was compared with other existing methods. Fig[6] represent the example ORL database image sets. The all above situations Tables [1,2,3, and 4] experiments values is clear to report this proposed method performance is good.

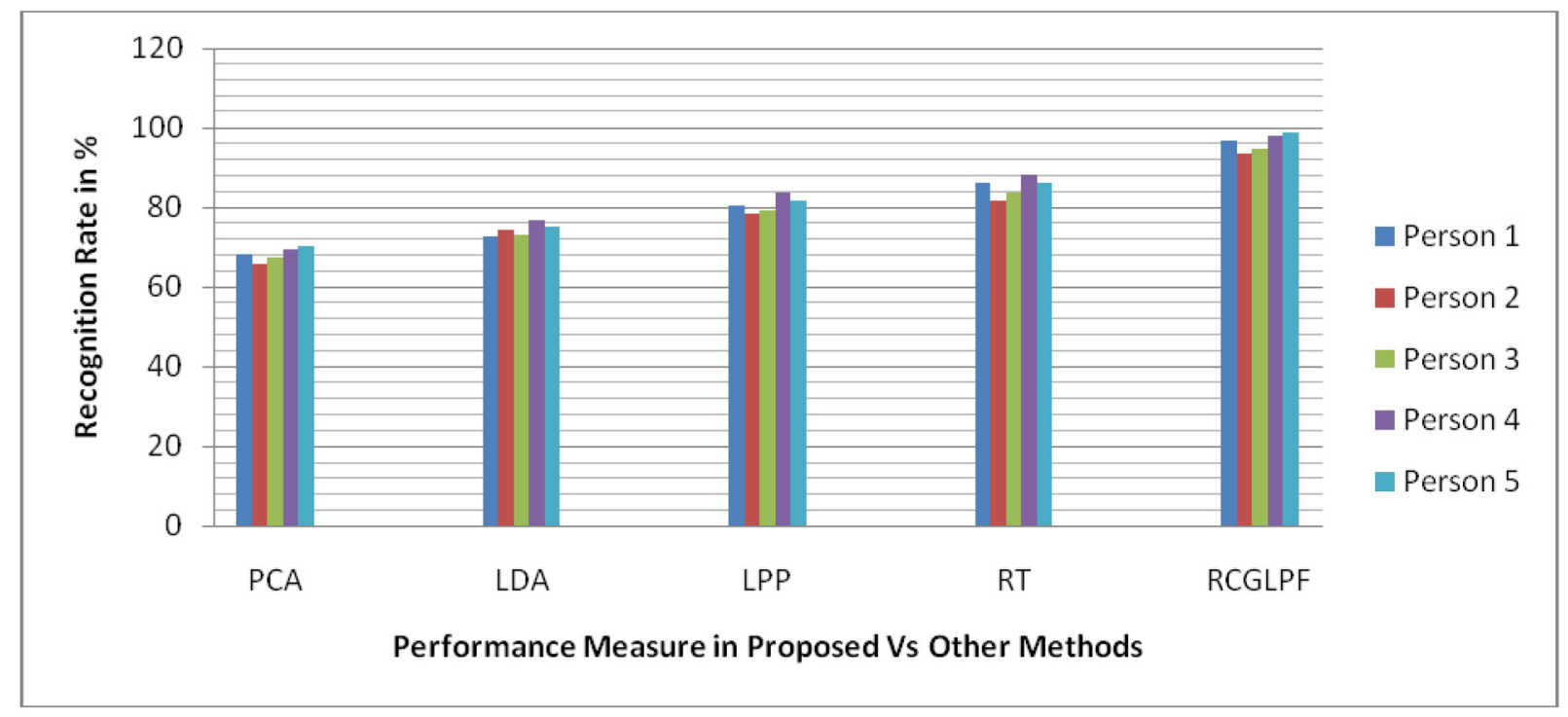

Figure 3. Recognition Rate for Proposed Vs Other Methods in Different Persons

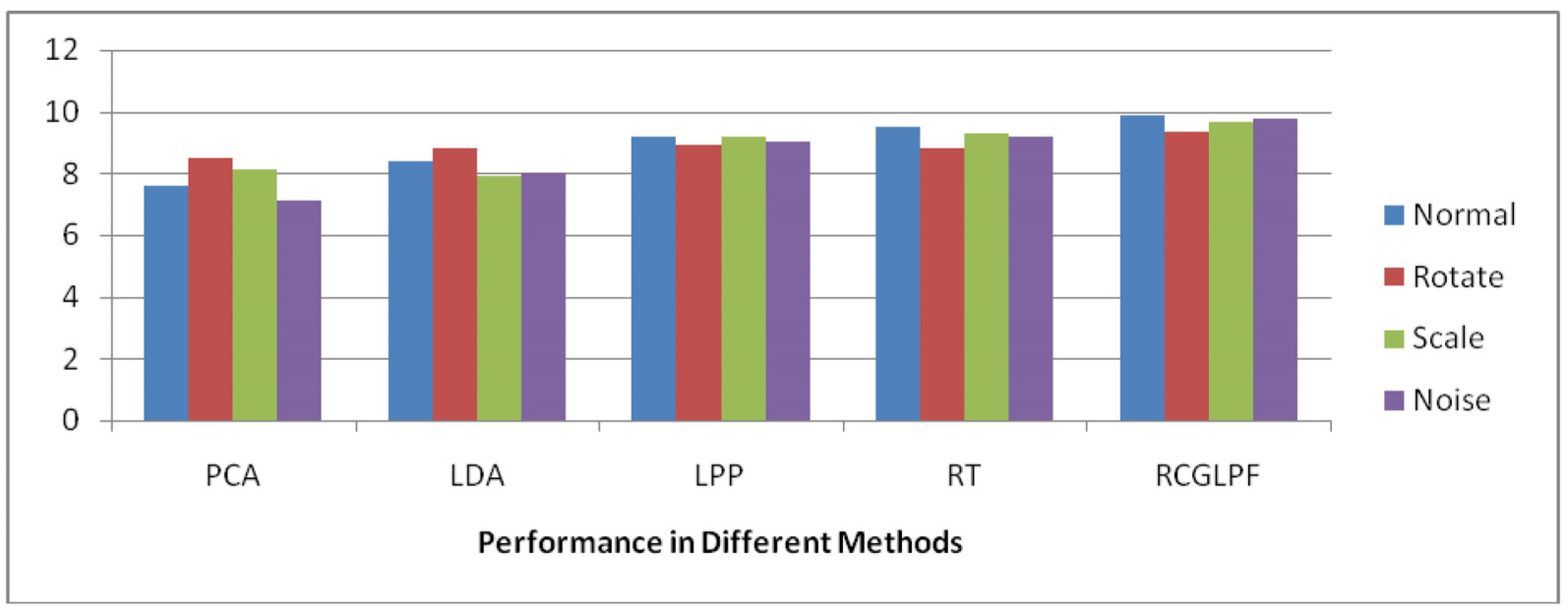

Figure 4. Performance of recognition chart in different query level in different met methods

\section{Conclusions}

Image Retrieval method has been described in this work. The main part of this work is LDA, LPP and PCA directly apply to the radon space relatively than faces. In this work radon transform used in this method was FFT based. The method was verified

\begin{tabular}{lllllll}
\hline 36 & Journal of Multimedia Processing and Technologies & Volume & 10 & Number 1 & March 2019 \\
\hline
\end{tabular}




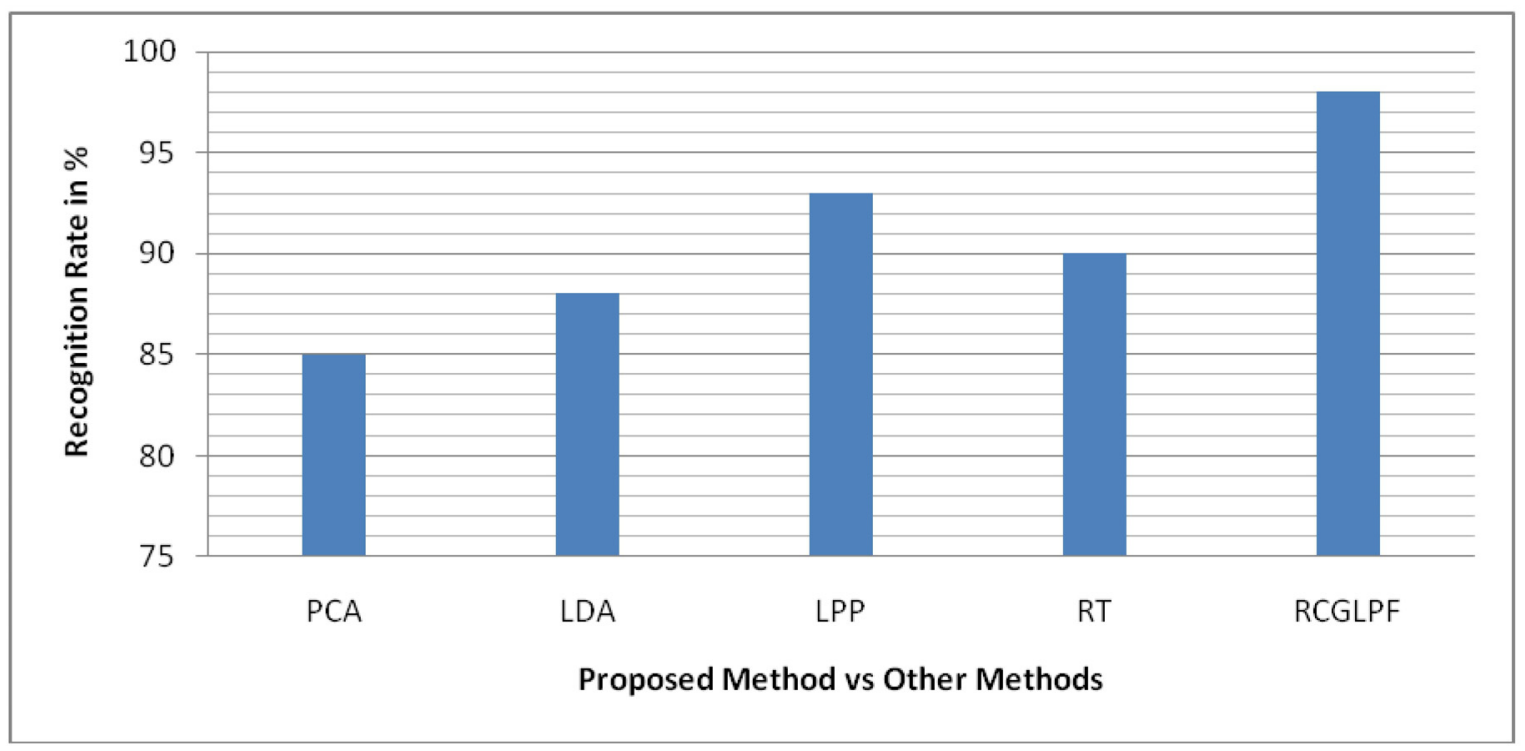

Figure 5. Performance of our proposed method recognition rate compared with other existing methods like Radon, LDA, LPP and PCA

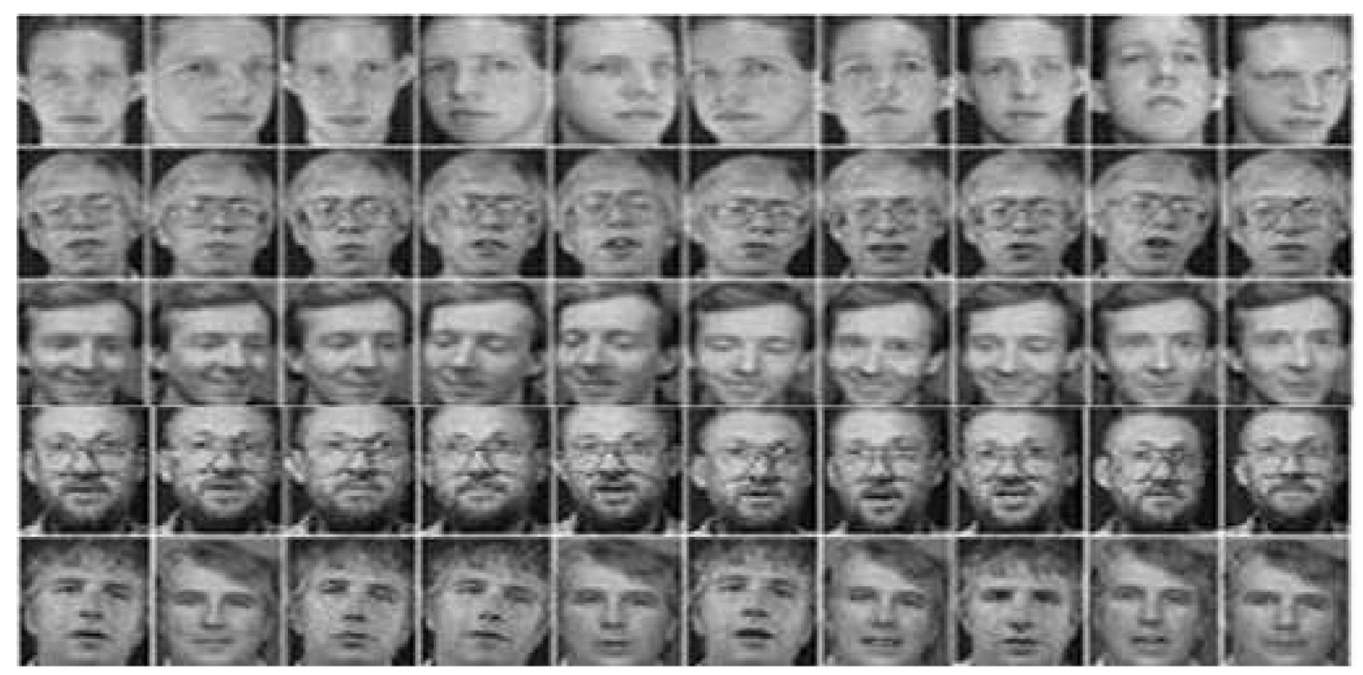

Figure 6. ORL Database

ORL Database contains different images of each of 40 distinct subjects. The best recognition rate $98.8 \%, 98.7 \%, 98.9 \%$ and $97.5 \%$ achieved by normal, rotated, scaled and noisy query environments.

Radon Combined Global and Local Preserving Features (RCGLPF) algorithm achieved in this method better than related works stated in the literature review section 2.

\section{References}

[1] Michel S lew, Sebe, Nicu., Djeraba, Chabane., Jain, Ramesh. (2006). Content-Based Multimedia Information Retrieval: State of the Art and Challenges, ACM Transactions on Multimedia Computing, Communications and Applications, 2 (1) 1-19.

[2] Ho Young Lee, Ho Keun Lee., Yeong Ho Ha. (2003). Spatial Color Descriptor for Image Retrieval and Video Segmentation, IEEE Transactions on Multimedia, 5 (3). 
[3] Quellec, Gwénolé., Lamard, Mathieu., Cazuguel, Guy. (2010). Adaptive Nonseparable Wavelet Transform via Lifting and its Application to Content-Based Image Retrieval, IEEE Transactions on Image Processing, 19 (1).

[4] Kokare, Manesh., Biswas, P. K., Chatterji, B. N. (2005). Texture Image Retrieval Using New Rotated Complex Wavelet Filters, IEEE Transactions on Systems Man, and Cybernetics_-PART B: Cybernetics, 35 (6).

[5] Wang, Hui Hui., Mohamad, Dzulkifli., Ismail, N. A. (2013). Semantic Gap in CBIR: Automatic Objects Spatial Relationships Semantic Extraction and Representation, International Journal of Image Processing (IJIP) 4 (3).

[6] Chiang, Te-Wei., Tsai, Tien-Wei. (2006). Content-Based Image Retrieval via the Multiresolution Wavelet Features of Interest, Journal of Information Technology and Applications, 1(3) 205-214.

[7] Quellec, Gwénolé., Lamard, Mathieu., Cazuguel, Guy. (2012). Fast Wavelet-Based Image Characterization for Highly Adaptive Image Retrieval, IEEE Transactions on Image Processsing, 21 (4).

[8] Gao, Yue., Wang, Meng., Zha, Zheng-Jun., Shen, Jialie., Li, Xuelong., Wu, Xindong. (2013). Visual-Textual Joint Relevance Learning for Tag-Based Social Image Search, IEEE Transations on Image Processing, 22(1).

[9] Zhang, Qianni., Izquierdo, Ebroul. (2013). Histology Image Retrieval in Optimized Multifeature Spaces, IEEE Journal of Biomedical and Health informatics, 17(1).

[10] Kekre, H. B., Mishra, Dhirendra. (2011). Content Based Image Retrieval Using Full Haar Sectorization, International Journal of Image Processing (IJIP), 5 (1).

[11] Chen, Yi-Chen., Challa S. Sastry, Vishal M. Patel, Jonathon Phillips, P., Chellappa, Rama. (2013). In-Plane Rotation and Scale Invariant Clustering Using Dictionaries, IEEE Transactions On Image Processing, 22 (6).

[12] Andrew, P., Papli'nski. (2010). Rotation Invariant Categorization of Visual Objects Using Radon Transform and Self-Organizing Modules, ICONIP 2010, Part II, LNCS 6444, p. 360-366.

[13] Andrew, P., Papli'nski. (2012). Rotation-Invariant Categorization of Colour Images using the Radon Transform, WCCI 2012 IEEE World Congress on Computational Intelligence, Brisbane, Australia.

[14] Hamid, M., Hasan, Waleed, A., Jouhar, A. L., Majid A., Alwan. (2012). Face Recognition Using Improved FFT Based Radon by PSO and PCA Techniques, International Journal of Image Processing (IJIP), 6(1).

[15] Jafari-Khouzani, Kourosh., Soltanian-Zadeh, Hamid. (2005). Rotation Invariant Multiresolution Texture Analysis Using Radon and Wavelet Transforms, IEEE Trans Image Process. 2005 June ; 14 (6) 783-795.

[16] Ahmed, Q., AL-Thahab. (2012). Image Recognition Using Combination of Multiwavelet and Radon Transforms with Neural Network, Journal of Babylon University/Engineering Sciences/ No.(1) Vol.(20).

[17] Image Noise, http://en.wikipedia.org/wiki/ Imagenoise

[18] NoiseModels, http://homepages.inf.ed.ac.uk/rbf/CVonline/LOCAL_COPIES/VELDHUIZEN/ node11.html

[19] Pu, Xiaorong., YI, Zhang., Fang, Zhongjie. (2008). Holistic and partial facial features fution by binary particle swarm optimization, Neural Computer \& Application 17. 481-488.

[20] Belhumeur, P. N., Hespanala, J. P., Kriegman, D. J. (1997). Eigenfaces vs. fisherfaces: recognition using class specific linear projection. IEEE Transactions on Pattern Analysis and Machine Intelligence, 19 (7) 711-720.

[21] Shanmugasundaram, Singaravelan., Dhanushkodi, Murugan. (2014). Content based Image retrieval using improved radon transform under various queries, World Applied Sciences Journal, 31 (11) 1952-1959.

[22] Belhumeur, P. N., Hespanha, J. P., Kriegman, D. J. (1997). Eigenfaces vs. Fisherfaces: recognitionusing class specific linear projection, IEEE Transactions on Pattern Analysis and Machine Intelligence, 19 (7) 711-720.

[23] Zolghadri-Jahomi, M., Valizadeh, M. R. (2006). A Proposed Query-Sensitive Similarity Measure, Iranian Journal of Science \& Technology, 30 (B2).

[24] Al-Khaffaf, H.S.M., Talib, A. Z., Abdul Salam, R. (2009). A Study on the effects of noise level, cleaning method, and vectorization software on the quality of vector data, Lecture Notes in Computer Science, 299-309.

[25] Singaravelan. S., Murugan. D., (2014). Image Retrieval Using Hybrid CRSFS Discriminate Pattern Selection Descriptor in Caltech 101 Database, World Applied Sciences Journal 32 (8) 1579-1586.

38 Journal of Multimedia Processing and Technologies Volume 10 Number 1 March 2019 
[27] Singaravelan, S. , Murugan, D., (2014). A Study of Content Based Image Retrieval Using Enhanced Radon Transform Space Features Set By Pcs And LDA Techniques, Informatologia. 47, 1, 12-19. 